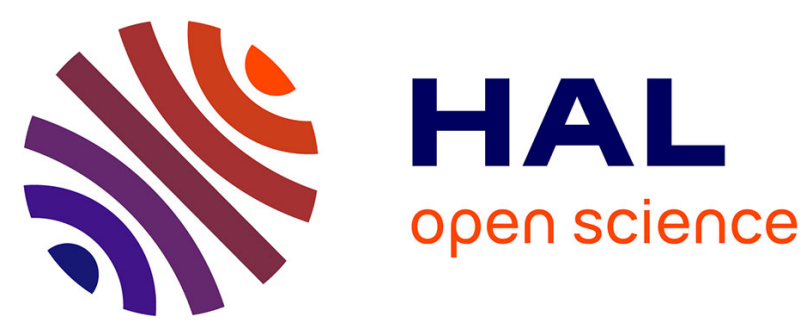

\title{
Theoretical Intrinsic Equivalent Magnetic Noise Evaluation for Magneto (Elasto) Electric Sensors Using Modulation Techniques
}

Xin Zhuang, Marc Lam Chok Sing, Christophe Dolabdjian, Peter Finkel, Jiefang Li, Dwight Viehland

\section{To cite this version:}

Xin Zhuang, Marc Lam Chok Sing, Christophe Dolabdjian, Peter Finkel, Jiefang Li, et al.. Theoretical Intrinsic Equivalent Magnetic Noise Evaluation for Magneto (Elasto) Electric Sensors Using Modulation Techniques. IEEE Sensors Journal, 2014, 14, pp.150-158. 10.1109/JSEN.2013.2274098 . hal-01061703

\section{HAL Id: hal-01061703 \\ https://hal.science/hal-01061703}

Submitted on 10 Jun 2015

HAL is a multi-disciplinary open access archive for the deposit and dissemination of scientific research documents, whether they are published or not. The documents may come from teaching and research institutions in France or abroad, or from public or private research centers.
L'archive ouverte pluridisciplinaire HAL, est destinée au dépôt et à la diffusion de documents scientifiques de niveau recherche, publiés ou non, émanant des établissements d'enseignement et de recherche français ou étrangers, des laboratoires publics ou privés. 


\title{
Theoretical intrinsic equivalent magnetic noise evaluation for Magneto(Elasto)Electric sensors using modulation techniques
}

\author{
X. Zhuang, M. Lam Chok Sing, C. Dolabdjian, P. Finkel, J. Li, and D. Viehland
}

\begin{abstract}
The equivalent magnetic noise of magnetostrictive-piezoelectric composite sensors, in the passive mode or when magnetic modulation techniques are used, has been investigated theoretically and compared to measurements. Several main noise sources and their contributions to the equivalent magnetic noise spectral density have been analyzed by using the fluctuation-dissipation theorem and modeled via Nyquist's noise-expression in the linear and non-linear regime. These theoretical analyses show that the mechanical loss, related to the interfriction of composites, appears as the dominant noise source for such magnetoelectric modulation techniques.
\end{abstract}

Index Terms-Magnetoelectric effects, Low-frequency noise modeling, magnetic signal modulation, Magnetic field sensing

\section{INTRODUCTION}

$\mathrm{S}_{\mathrm{p}}^{\mathrm{tr}}$ train-induced magnetoelectric (ME) effect has been proposed for magnetic field sensing as a replacement of single-phase materials with generally weak magnetoelectric couplings during the recent years. The induced electric polarization in the piezoelectric layer is linearly coupled to the magnetic field via an inter-mediated deformation between the magnetostrictive and piezoelectric layers. Prior efforts have been devoted to increasing the ME coupling in hetero-structural laminates, which is regarded as a fundamental problematic in condensed material physics, in order to satisfy applications for such devices. Several materials with high effective magnetostrictive coefficients (such as Terfenol-D, Nickel, Metglas) and high piezoelectric coefficients (such as PZT, PMN-PT) have been chosen for enhancing the ME effect [1-5]. Besides, the geometry of the ME laminates and the thickness ratio between magnetostrictive and piezoelectric layers have also been investigated to optimize the ME coupling [6]. To date, at quasi-static frequencies, the highest ME coefficient has been reported for a sandwiched structural laminate using two dimensional Metglas thin layers and one dimensional PMN-PT

Manuscript received Mai 13, 2013.

X. Zhuang, M. Lam Chok Sing and C. Dolabdjian are with the Groupe de Recherche en Informatique, Image, Automatique et Instrumentation de Caen (GREYC), CNRS UMR 6072 - ENSICAEN and the University of Caen, 14050 Caen Cedex, France (e-mail: xzhuang11@yahoo.fr).

F. Peter is with the Naval Undersea Warfare Center, Newport, Rhode Island 02840, USA

J. Li and D. Viehland are with the Materials Science and Engineering Department, Virginia Tech, Virginia 24061, USA. fibers, around $52(\mathrm{~V} / \mathrm{cm}) / \mathrm{Oe}$. For mechanical resonant detection, alternative thin film composites with tip-mass have demonstrated colossal ME voltage coefficients of about $1800(\mathrm{~V} / \mathrm{cm}) / \mathrm{Oe}$ when working at the first mechanical bending resonance [7]. These enhancements of the ME coupling make such devices more attractive for engineering applications of magnetic field sensing, as magnetic sensors. The limit of detection (LOD) is an important parameter which characterizes the performance of a magnetic field sensing element for applications. For a magnetic sensor based on ME laminates, this limit is influenced by both the magnetic field sensitivity and the noise level in the composites [8].

The equivalent magnetic noise (EMN) spectral density has been used for determining the LOD of magnetic sensors based on ME laminates [9]. The contribution of all the noise sources measured at the output terminal of the sensor can be expressed as an input magnetic noise source at the input terminal, via the magnetic field sensing transfer coefficient. So far, the lowest reported EMN spectral density level is about $5 \mathrm{pT} / \sqrt{\mathrm{Hz}}$ at $1 \mathrm{~Hz}$ for sandwiched structural laminates using two dimensional Metglas thin layers and one dimensional PMN-PT fibers [10]. Electrical noise sources in the piezoelectric layer have been proved to be the principle noise sources that give the dominant contribution to the EMN spectral density level [11-14]. The first type of noise source in the piezoelectric layer is a Johnson noise that results from the free electric charge random motions which are related, for example, to the motion of oxygen vacancies [15]. Dielectric loss noise is another noise source related to the electric dissipation from electric polar domain wall motion [16]. Both of these two types of noise can be considered as thermo-electric noise sources, according to the fluctuation-dissipation theory. However, to lower the EMN spectral density level by orders in magnitude is a difficult task if considering only the current piezoelectric material fabrication techniques.

In order to avoid this limitation from piezoelectric materials, several research groups have tried to use the magneto-electric cross modulation technique for lowering the EMN spectral density level with a hope of enhancing the performance in magnetic field sensing by using ME composites [17-19]. This idea is feasible because of the fact that both the Johnson noise level and the dielectric loss noise level decrease as a function of frequency. The low frequency magnetic field to be sensed can modulate an excitation carrier which is applied on the ME 
composite at a high frequency, particularly around the first mechanical resonant frequency. The low frequency output signals can be separated from the carrier by means of classical demodulation techniques. Thus, the only expected noise is the one around the frequency of the excitation carriers. The noise contribution from the low frequency thermo-electric dissipations should be definitely avoided. However, the measurements have shown that a non-negligible noise is distributed around the excitation carrier and is presented as a $1 / f$ noise after the demodulation process [20]. In order to enhance the performance with magnetoelectric cross modulation, the origin of the near-carrier noise and the noise transmission for diverse noise sources need to be investigated.

A magnetostrictive-piezoelectric composite response contains several intrinsic noise sources [21-25] associated to the energy losses such as thermo-electronic noise, thermo-mechanical noise, thermo-magnetic noise, etc. These intrinsic noise sources have their own contributions at the output terminals via their linear or non-linear transfer functions. Unlike the traditional passive detection mode, if a high frequency magnetic or electric signal is applied to the magnetoelectric composite as an excitation signal, the latter can be amplitude-modulated by the applied low frequency magnetic signal to be detected via any magnetic nonlinearities of the composite.

In this paper, we analyze the intrinsic noise sources in sandwich type ME composites, by using the fluctuation-dissipation theorem with Nyquist's expression for noise. Linear and non-linear transfer functions for the applied magnetic field and for other possible noise sources have been investigated for a better understanding of the performances of a ME composite operating under passive and active modulation methods, respectively. The terms "passive" and "active" are utilized to distinguish between the classical detection mode and the ME modulation technique.

\section{INTRINSIC NOISE SOURCES}

\section{A. Description for sensor modeling}

A ME hetero-structure laminate composite consists of three layers, namely a magnetostrictive layer, a piezoelectric layer and an elastic inter-mediate layer, respectively. The magnetostrictive layer, also called the passive layer, serves for sensing the magnetic field and generating an elastic deformation. The inter-mediate layer is used for coupling the magnetostrictive and piezoelectric layers. Any mechanical deformation can be transferred via this layer to produce a strain in the piezoelectric layer which can induce ME effects. The piezoelectric material serves as a so-called active layer in the ME composite, generating electric signals via the deformation from the magnetostrictive layer [26]. Electrodes are often integrated across this layer to recover the electric signals. Neglecting the influence of the elastic inter-mediate layer, a simple schematic diagram of a ME composite working in longitudinally magnetic and electric polarization conditions, as a piezoelectric layer sandwiched between two magnetostrictive layers, is shown in Fig. 1. The parameters $E, D, H, B, T, S$ are the electric field, electric displacement, magnetic field, magnetic induction, stress and strain in the longitudinal direction (noted "3"), respectively. The thickness and width direction are defined by " 2 " and "1", respectively. The geometrical dimensions are $l \times w \times t_{p}$ for the piezoelectric layer and $l \times w \times t_{m} / 2$ for either of the magnetostrictive layers. The induced electric voltage $V$ or current $I$ is collected along the longitudinal direction by means of the electrodes.

In an energy storage system, any damping induces corresponding dissipations $[27,28]$. The fluctuationdissipation theorem states that each type of dissipation produces random fluctuations. These random fluctuations in an enclosed system are usually represented as noise sources. With Nyquist's noise expression, the noise sources are directly related to the real part of the damping term in the damping system. A ME composite has magnetic, mechanical and electrical properties because of the magnetostrictive piezoelectric materials that are used and of the mechanical-mediated transferring method. Thus, the intrinsic noise sources can be investigated by analyzing the magnetic, mechanical and electric dissipations.

\section{B. Electric noise sources}

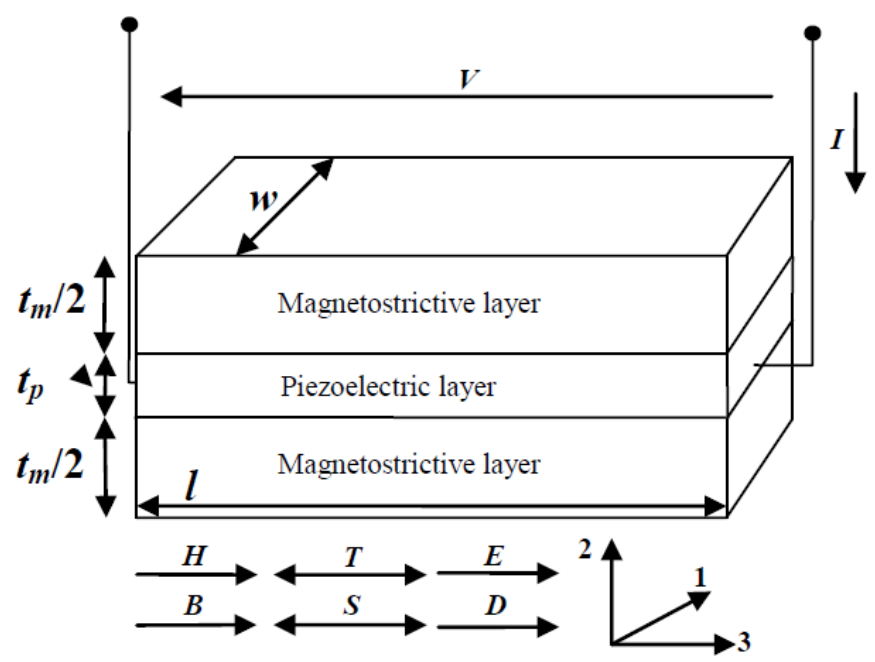

Fig. 1. Sketch view of the laminated magnetoelectric composite model. $w, t_{m}, t_{p}, V, I, H, B, E, D T, S$, are the width, of sensors, the thicknesses of the magnetostrictive and piezoelectric layers, the voltage and the current induced by the piezoelectric layer, the magnetic field and the magnetic induction, the electric field and electric induction, the stress and the strain, respectively.

In the piezoelectric layer of a ME composite, the thermoelectric noise induced by the electric dissipation consists of electric conduction loss and dielectric loss noise sources. Johnson noise is due to the random fluctuation of free electric charges such as oxygen vacancies. This free charge motion is related to the electrical resistance $R$ which is a noisy element and the corresponding Johnson current noise can be written via the Nyquist's noise expression as

$$
i_{n_{-} R}(f)=\sqrt{\frac{4 k_{B} T}{R}}
$$

where $k_{B}$ is the Boltzmann constant and $T$ is the absolute temperature in Kelvin. 
The dielectric loss noise in the piezoelectric layer is induced by the random fluctuations of domain wall motions. Both $180^{\circ}$ and non- $180^{\circ}$ electric polar domain wall motions can contribute to the dielectric loss noise source where the $180^{\circ}$ electric domain wall motion is dominant [29-32]. This noise is related to the loss factor $\tan \left(\delta_{\text {elec }}\right)$ which is defined by the ratio of $\varepsilon$ " the imaginary part and $\varepsilon^{\prime}$ the real part of the dielectric constant: i.e. $\tan \left(\delta_{\text {elec }}\right)=\varepsilon^{\prime \prime} / \varepsilon^{\prime}$. The noisy element in this case, is the electric capacitance, $C$, of the piezoelectric layer. By using Nyquist's expression, the noise spectral density can be written as

$$
i_{n_{-} C}(f)=\sqrt{4 k_{B} T 2 \pi f C \tan \left(\delta_{\text {elec }}\right)}
$$

where $f$ is the frequency.

The first noise source (1) is white whereas the second (2) shows a $\sqrt{ } f$ dependence. Thus, by using a charge amplifier, these two noise sources are integrated and lead to respectively a $1 / f$ and $1 / \sqrt{ } f$ frequency dependence in the noise spectral density curves, which dominate at low frequencies and thus, determine the detection limit $[14,32]$.

\section{Mechanical noise sources}

Mechanical dissipation exists in both the magnetostrictive and piezoelectric layers of a ME laminate [33,34]. This dissipation generates a thermo-mechanical noise source having two main origins: the viscous loss and interfriction which are related to the dynamic mass motion and the non $-180^{\circ}$ domain wall motions respectively [16]. The random fluctuation of the dynamic mass motion is related to the mechanical resistance, $R_{\text {mech }}$. The latter describes the relationship between the force and the vibration speed in the oscillation system. This random force noise source can be expressed as a Nyquist's noise formula

$$
f_{n_{-} R_{\text {mech }}}(f)=\sqrt{4 k_{B} T R_{\text {mech }}} \text {. }
$$

By using the expression of $R_{\text {mech }}=2 \pi f_{0} \mathrm{~m} / Q$ [11], (3) can be rewritten as

$$
f_{n_{-} R_{\text {mech }}}(f)=\sqrt{4 k_{B} \operatorname{Tm} 2 \pi f_{0} / Q}
$$

where $m$ is the mass of the laminate, $f_{0}$ is the first resonance frequency and $Q$ is the damping coefficient. In certain ME composites, $Q$ is determined by the damping quality in the bonding layers.

The interfriction loss, related to the non- $180^{\circ}$ domain wall motion of both magnetostrictive and piezoelectric layers can be represented by a noise source resulting from a mechanical capacitance. For a longitudinal motion of the ME composite, this mechanical capacitance defines the relation between force and deformation as

$$
C_{\text {mech }}=s_{33} l / w t_{\text {lam }}
$$

where $s_{33}$ and $t_{\text {lam }}$ are the elastic compliance constant and the total thickness of the ME laminate, respectively. The mechanical compliance represents the relation between mechanical force and deformation. Actually, there exists a time delay between these two mechanical parameters, which can be expressed in a complex form as $s=s^{\prime}\left(1+j \tan \left(\delta_{\text {mech }}\right)\right)$ where the mechanical loss factor $\tan \left(\delta_{\text {elec }}\right)=\varepsilon " / \varepsilon$ ' is the ratio between imaginary and real part of the composite flexibility coefficient.

The real part of the mechanical impedance $Z_{\text {mech }}=1 / j 2 \pi f C_{\text {mech }}$ can be written as

$$
\operatorname{Re}\left\{Z_{\text {mech }}\right\}=\frac{\tan \left(\delta_{\text {mech }}\right)}{2 \pi f C_{\text {mech }}} .
$$

With the help of Nyquist's expression, the mechanical loss noise can be represented as a force noise source given by

$$
f_{n_{-} C_{\text {mech }}}(f)=\sqrt{4 k_{B} T \operatorname{Re}\left\{Z_{\text {mech }}\right\}}=\sqrt{4 k_{B} T \tan \left(\delta_{\text {mech }}\right) / 2 \pi f C_{\text {mech }}} .
$$

Considering the ME laminate as a Kelvin-Voigt material, the displacement noise formula can obtained from the total mechanical force, $f_{n}^{2}=f_{n_{-} R_{\text {meth }}}^{2}+f_{n_{-} C_{\text {meth }}}^{2}$, and the mechanical impedance $Z_{\text {mech }}$, as

$$
\begin{aligned}
x_{n}(f) & =\frac{f_{n}(f)}{\left|2 \pi f Z_{\text {mech }}\right|} \\
& =\sqrt{4 k_{B} T\left(R_{\text {mech }} C_{\text {mech }}^{2}+\frac{C_{\text {mech }} \tan \left(\delta_{\text {mech }}\right)}{2 \pi f}\right)} .
\end{aligned}
$$

We notice that the displacement noise consists of a white noise part due to the viscous loss and a $1 / f$ dependant term due to interfriction loss. This random motion can be directly transferred to the output terminal of the ME laminate as an electric noise via the piezoelectric layer.

\section{Magnetic noise sources}

In the magnetostrictive material, the motions of the two kinds of domain walls $\left(180^{\circ}\right.$ and non- $\left.180^{\circ}\right)$ are quite different. Temperature-induced domain wall motion results in a random magnetic fluctuation which can be considered as a thermo-magnetic noise source in the magnetic layers [35, 36]. Both $180^{\circ}$ and non- $180^{\circ}$ domain walls motion induce a random fluctuation of the magnetization as a thermo-magnetic noise source in the magnetostrictive layer. Moreover, the $180^{\circ}$ domain walls motions produce the dominant contribution to this noise source compared to the non- $180^{\circ}$ domain wall motion. Since the passage of a $180^{\circ}$ domain wall through a certain region reverses the magnetization of that region, we conclude that $180^{\circ}$ wall motions do not produce a net magnetostrictive change. Thus, only the non $-180^{\circ}$ domain wall motions will result in net mechanical random fluctuations that are coupled to the magnetic random fluctuations for this type of domain wall motions. 
In general, the non- $180^{\circ}$ domain wall motions also produce a thermo-mechanical noise source in the magnetostrictive layers. This can be described via the interfriction in the magnetostrictive materials by using the mechanical loss factor which is defined as the ratio between the imaginary and real parts of the elastic coefficients. This effect can be regarded as a mechanical delay between strains and stresses [24, 32,37]. Viscous losses are another mechanical dissipations in ME hetero-structure composites. This type of loss occurs in all the three layers in the ME hetero-structure, which can be explained by the damping in the materials resulting from their dynamic mass. In general, the thermo-mechanical, which is related to the viscous loss, can be described by the mechanical quality factor. We consider that the thermo-magnetic random fluctuations have a negligible contribution to the piezoelectric layers without induced strain.

\section{SIGNAL AND NOISE}

\section{A. Equations for the sensor model}

From the linear magnetostrictive and piezoelectric constitutive equations [14], we can obtain the relations between the magnetic field, the electric voltage and the current for a longitudinal magnetization and polarization mode for a $\mathrm{ME}$ composite [1] under free-free boundary mechanical conditions, as shown in Fig. 1. This can be expressed as

$$
\left\{\begin{array}{l}
\left(v_{1}+v_{2}\right) Z_{\text {mech }}+\varphi_{m} H+\varphi_{p} V=0 \\
\left(v_{1}+v_{2}\right) \varphi_{p}+j 2 \pi f C V+I=0 \\
F=\left(v_{1}+v_{2}\right) Z_{\text {mech }}
\end{array}\right.
$$

where $v_{1}$ and $v_{2}$ are the vibration speeds at the two free ends of the composite along the longitudinal direction, $F$ is the applied force. $\quad s_{33}\left(=\left((1-n) / s_{33, p}+n / s_{33, m}\right)^{-1}\right)$ is the mean

flexibility coefficient of the magnetostrictive and piezoelectric layer with a thickness ratio $n=t_{m} / t_{\text {lam }}$, the magnetic and electric coupling coefficients are $\varphi_{m}=\frac{d_{33, m} t_{m} w}{s_{33, m}} \quad$ and $\quad \varphi_{p}=\frac{d_{33, p} t_{p} w}{s_{33, p} l} \quad, \quad$ respectively. $C\left(=\varepsilon w t_{p} / l\right)$ is the electronic capacitance of the piezoelectric layer, and $H, V$ and $I$ are the applied magnetic field, the electric voltage and the current across the electrode, respectively.

As we know, the response of a ME laminate sensor is not always linear. Considering the first order of the magnetic nonlinearity, the magnetic coupling coefficient can be defined as

$$
\varphi_{m}^{N L}=\frac{d_{33, m}^{N L} t_{m} w}{s_{33, m}}
$$

where $d_{33, m}^{N L}$ term represents the first order nonlinear magnetostrictive coefficient.
Taking into account both the magnetic nonlinearity $\eta\left(=d_{33, m}^{N L} / d_{33, m}\right)$ and the mechanical nonlinearity $\kappa\left(=s_{33}^{N L} / s_{33}\right)$ and the piezoelectric nonlinearity $\zeta=\frac{d_{33, p}^{N L}}{d_{33, p}}$,

(9) can be rewritten in its nonlinear form as

$$
\left\{\begin{array}{l}
F+\kappa F^{2}+\varphi_{m}(1+\eta H) H+\varphi_{p}(1+\zeta V) V=0 \\
\frac{F+\kappa F^{2}}{Z_{\text {mech }}} \varphi_{p}+j 2 \pi f C V+I=0
\end{array} .\right.
$$

\section{B. ME charge coefficient}

In order to obtain the magnetic signal transfer function, we can find the relation between the current and the magnetic field under a short circuit condition $(V=0)$ from (11). This yields

$$
\varphi_{m}(1+\eta H) H=\frac{Z_{m e c h}}{\varphi_{p}} I
$$

The magnetic current transfer function, defined by the current magnetoelectric coefficient, can be written by means of the derivative of (12) for a magnetic field $H$. This gives

$$
\alpha_{M E}^{I}=\left|\frac{\partial I}{\partial H}\right|=\frac{\varphi_{m} \varphi_{p}}{Z_{\text {mech }}}+2 \frac{\eta \varphi_{m} \varphi_{p} H}{Z_{\text {mech }}} .
$$

The first and second terms in (13) are the linear and the non-linear magneto-elastic coupling coefficients, respectively. We find that the nonlinear ME coefficient depends on the magnetic nonlinearity and the applied magnetic field on the composite. The ME charge coefficient can then be expressed as

$$
\alpha_{M E}^{Q}=\frac{1}{2 \pi f}\left|\frac{\partial I}{\partial H}\right|=\frac{\varphi_{m} \varphi_{p}}{2 \pi f Z_{\text {mech }}}+\frac{\eta \varphi_{m} \varphi_{p} H}{\pi f Z_{\text {mech }}} .
$$

\section{Charge noise}

As we discussed in the above section, the thermo-magnetic noise in the magnetostrictive layer, due to $180^{\circ}$ domain wall motion, does not induce any deformation on the laminate. Therefore, this noise source in the magnetostrictive layer has no output noise contribution because it does not result in a net deformation onto the piezoelectric layer. In other words, the transfer function of the thermo-magnetic noise is considered to be null. The electric loss induced Johnson noise and the dielectric loss noise from the random motion of the free electric charges and from the electric domain wall motions contribute to the output terminal of the ME laminate, directly. This means that the transfer function of such noise sources is equal to 1 . However, the mechanical loss consists of the viscous loss and the interfriction loss, both in the magnetostrictive and piezoelectric layers. In this case, the transfer function depends on the piezoelectric coefficient and on the mechanical impedance. 
Under the short circuit condition $(V=0)$, the second equation in (11) can be rewritten as

$$
\frac{F+\kappa F^{2}}{Z_{\text {mech }}} \varphi_{p}+I=0 .
$$

By taking the derivative of (15) with respect to the mechanical force, the transfer function for the force $F$ to the electric current $I$ can be obtained as

$$
\left|\frac{\partial I}{\partial F}\right|=\frac{\varphi_{p}}{Z_{\text {mech }}}+\frac{2 \kappa \varphi_{p} F}{Z_{\text {mech }}} .
$$

Generally speaking, the magnetic induced force includes any magnetic nonlinearities and it can be written as $F=\varphi_{m} H+\varphi_{m}^{N L} H^{2}+\ldots$ The first term, representing the linear response, is the predominant term. Therefore, by choosing a linear relationship between the mechanical force and the magnetic signal, $F=\varphi_{m} H$, and assuming there is no loss, (16) becomes

$$
\left|\frac{\partial I}{\partial F}\right|=\frac{\varphi_{p}}{Z_{m e c h}}+\frac{2 \kappa \varphi_{p} \varphi_{m} H}{Z_{m e c h}} .
$$

Thus, the electric current noise spectral density due to the mechanical noise force can be written as in

$$
i_{n}(f)=\frac{\varphi_{p}}{Z_{\text {mech }}} f_{n}+\frac{2 \kappa \varphi_{p} \varphi_{m} H}{Z_{\text {mech }}} f_{n} .
$$

By using the mechanical noise formula from (3) and (7), the output electric charge noise can be written as

$$
\begin{aligned}
q_{n}(f) & =\varphi_{p} \sqrt{\frac{4 k_{B} T \tan \left(\delta_{\text {mech }}\right) C_{\text {mech }}}{2 \pi f}+4 k_{B} T R_{\text {mech }} C_{\text {mech }}^{2}} \\
& +2 \kappa \varphi_{p} \varphi_{m} H \sqrt{\frac{4 k_{B} T \tan \left(\delta_{\text {mech }}\right) C_{\text {mech }}}{2 \pi f}+4 k_{B} T R_{\text {mech }} C_{\text {mech }}^{2}}
\end{aligned}
$$

We thus find that without any magnetic excitation signal, the output electric charge noise is linearly distributed at quasi-static frequencies. However, if driven by a magnetic carrier, the low frequency mechanical noise can modulate the excitation signal and it will be distributed around the drive carrier frequency as we shall detail, hereafter.

\section{EQUIVALENT MAGNETIC NOISE}

\section{A. Equivalent Magnetic Noise for Passive Sensing}

For passive sensing, the linear transfer function is the dominant ME sensitivity term and the electric and mechanical original noise sources have their own contributions to the total noise floor. Since the intrinsic thermo-magnetic noise source does not have any contribution to the total output electric noise, we take into account only a) the thermo-electric noise due to the

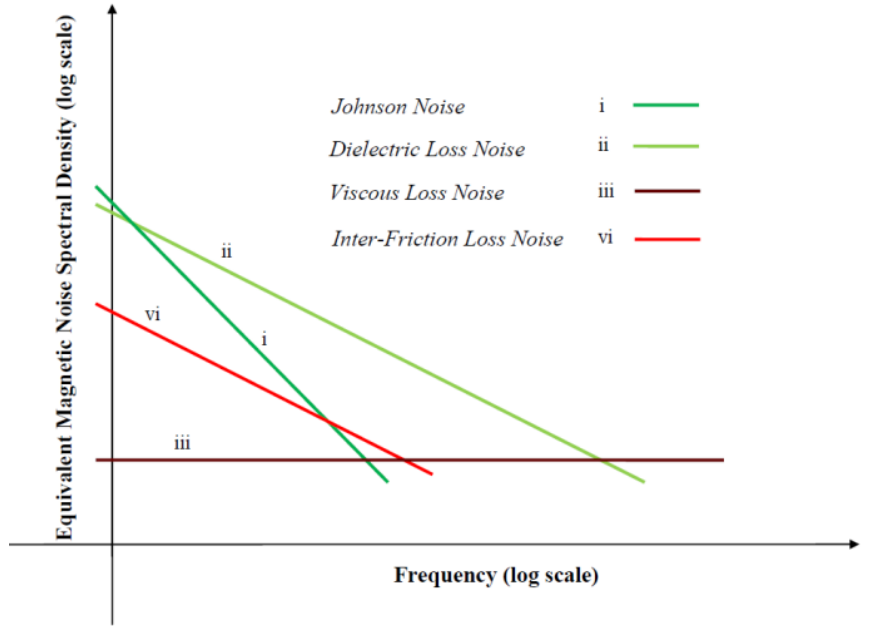

Fig. 2. Equivalent magnetic noise spectral densities as a function of frequency for the intrinsic noise sources in a magnetoelectric sensor (cf. (22)).

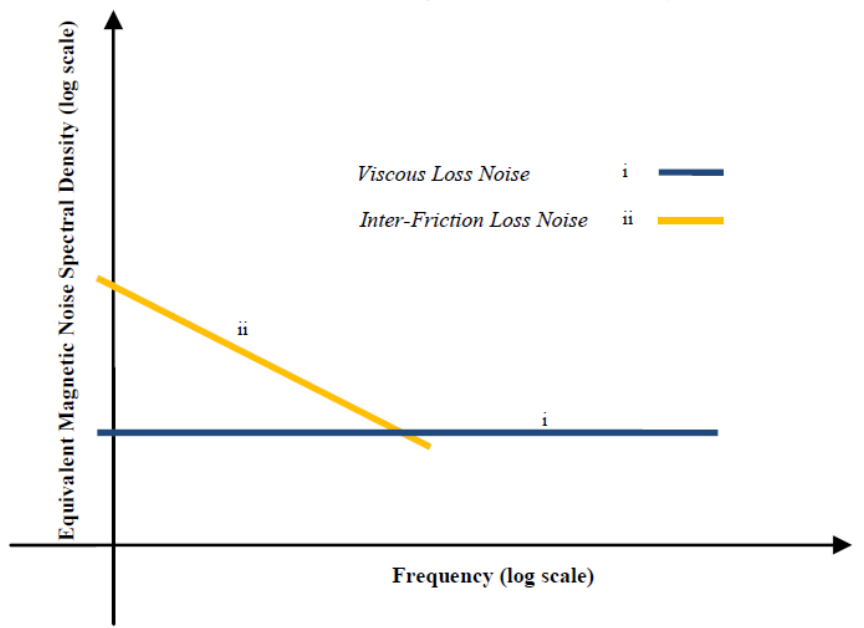

Fig. 3. Expected equivalent magnetic noise spectral density curve of a $\mathrm{ME}$ sensor appearing at low frequencies when modulation techniques are used, (cf. (29)). This apparent magnetic noise is due to the mechanical noise sources in the laminate.

$180^{\circ}$ domain wall motion and free charge random motion, b) thermo-mechanical noise sources due to the non- $180^{\circ}$ domain wall motion and dynamic mass random motion for analysis. At low frequencies and in the passive working mode, the total output electric charge noise spectral formula provided by the thermo-electric and thermo-mechanical random fluctuations can be calculated. It yields

$$
\begin{aligned}
q_{n}^{2}(f) & =4 k_{B} T\left(\frac{1}{(2 \pi f)^{2} R}+\frac{C \tan \left(\delta_{\text {elec }}\right)}{2 \pi f}\right. \\
& \left.+\frac{\varphi_{p}^{2} C_{\text {mech }} \tan \left(\delta_{\text {mech }}\right)}{2 \pi f}+\left(C_{\text {mech }} \varphi_{p}\right)^{2} R_{\text {mech }}\right)
\end{aligned}
$$

It can be seen that the four terms in (20) correspond to the electric conduction loss, the dielectric loss, the mechanical interfriction loss and the viscous loss and have a $1 / f, 1 / \sqrt{ } f, 1 / \sqrt{ } f$ and white noise density spectrum, respectively. The equivalent noise spectral curves are seen in Fig. 2.

At low frequencies, the linear part of the ME charge coefficient represents the main contribution to the ME 
coefficient. By replacing the mechanical impedance by $Z_{\text {mech }}=1 /\left(2 \pi f C_{\text {mech }}\right)$, the charge coefficient at low frequencies can be deduced as

$$
\alpha_{M E}^{Q}=\varphi_{m} \varphi_{p} C_{m e c h} .
$$

By using the noise formula (20) and the sensitivity expression (21), we can find the equivalent magnetic noise power expression for the low frequency response as

$$
\begin{aligned}
b_{n}^{2}(f) & =\frac{\mu_{0}^{2} q_{n}^{2}(f)}{\left(\alpha_{M E}^{Q}\right)^{2}} \\
= & \mu_{0}^{2} 4 k_{B} T\left(\frac{\tan \left(\delta_{\text {mech }}\right)}{2 \pi f \varphi_{m}^{2} C_{\text {mech }}}+\frac{R_{\text {mech }}}{\varphi_{m}^{2}}\right. \\
& \left.+\frac{1}{(2 \pi f)^{2} R\left(\varphi_{m} \varphi_{p} C_{\text {mech }}\right)^{2}}+\frac{C \tan \left(\delta_{\text {elec }}\right)}{2 \pi f\left(\varphi_{m} \varphi_{p} C_{\text {mech }}\right)^{2}}\right)
\end{aligned}
$$

By using the physical parameters of the sensor and the relation $R=l \rho_{\text {elec }} / t_{p} w$ and $f_{0}=(1 / 2 l) \sqrt{1 / \rho s},(22)$ becomes

$$
\begin{aligned}
b_{n}^{2}(f) & =\left(\frac{\mu_{0} s_{33, m} s_{33, p}}{n \omega d_{33, m} d_{33, p} s_{33}}\right)^{2} \frac{4 k_{B} T}{\rho_{\text {elec }} V_{p}} \\
& +\left(\frac{\mu_{0} s_{33, m} s_{33, p}}{n d_{33, m} d_{33, p} s_{33}}\right)^{2} \frac{2 k_{B} T \varepsilon_{33}^{S} \tan \left(\delta_{\text {elec }}\right)}{\pi f V_{p}} \\
& +\left(\frac{\mu_{0} s_{33, m}}{n d_{33, m}}\right)^{2} \frac{2 k_{B} T \tan \left(\delta_{\text {mech }}\right)}{\pi f s_{33} V_{\text {lam }}} \\
& +\left(\frac{\mu_{0} s_{33, m}}{n d_{33, m}}\right)^{2} \frac{4 k_{B} T \pi \sqrt{\rho / s}}{w t Q}
\end{aligned}
$$

where $\rho_{\text {elec }}$ is the resistivity, $V_{p}$ the volume of the piezoelectric layer, $V_{\text {lam }}$ the volume of ME composite and $\rho$ the mean value of the volume density for the composite.

\section{B. Equivalent Magnetic Noise when using modulation techniques}

By using a frequency shift modulation technique, the ME composite is excited by an external sinusoidal signal. This can be a harmonic magnetic field, an electric field or a mechanical vibration of high frequency. An applied low frequency magnetic signal modulates the excitation signal via the nonlinearity of the composite. This is revealed as two side-band signal peaks around the excitation signal frequency in the spectrogram similar to a classical amplitude modulation (AM). Electric noise sources in the piezoelectric layer are directly observed at the output terminal of the ME composite. These electric noise sources are not influenced by any nonlinearity of the ME composite. Thus, there is no noise contribution from these noise sources using ME modulation techniques. The noise source related to the thermo-magnetic loss does not result in a mechanical random fluctuation in the ME composite. Therefore, it does not make any noise contribution either for the classical passive mode or for the frequency modulation technique(s). The mechanical noise sources related to the thermo-mechanical loss in the magnetostrictive and piezoelectric layers can modulate the excitation deformation and give a corresponding noise output when using a classical demodulation. This noise source is considered as the dominant noise source for the frequency modulation technique.

Replacing the magnetic excitation $H$ by a harmonic magnetic signal of amplitude $H_{0}$ at a frequency $f_{0}$, we can express the nonlinear magnetoelectric transfer function as

$$
\alpha_{N L M E}^{Q}=\frac{\eta \varphi_{m} \varphi_{p} H_{0}}{2 \pi f Z_{\text {mech }}} *\left(\delta\left(f-f_{0}\right)+\delta\left(f+f_{0}\right)\right) .
$$

Similarly, the thermo-mechanical noise distributed around the excitation signal carrier can be written as

$$
\begin{aligned}
& q_{n_{-} \bmod }(f)=\left(\delta\left(f+f_{0}\right)+\delta\left(f-f_{0}\right)\right) * \frac{\kappa \varphi_{p} \varphi_{m} H_{0}}{2 \pi f Z_{\text {mech }}} \\
& \sqrt{\frac{4 k_{B} T \tan \left(\delta_{\text {mech }}\right)}{2 \pi f C_{\text {mech }}}+4 k_{B} T R_{\text {mech }}}
\end{aligned}
$$

By using a synchronous detector, $\operatorname{Cos}\left(2 \pi f_{0} t\right)$, the magnetic transfer function at low frequencies is given by

$$
\alpha_{N L M E}^{Q}=\frac{\eta \varphi_{m} \varphi_{p} H_{0}}{2 \pi f Z_{\text {mech }}} .
$$

The thermo-mechanical noise expression at low frequencies can be written as

$$
\begin{aligned}
q_{n_{-} \text {demod }}(f) & =\frac{\kappa \varphi_{p} \varphi_{m} H_{0}}{2 \pi f Z_{\text {mech }}} \\
& \sqrt{\frac{4 k_{B} T \tan \left(\delta_{\text {mech }}\right)}{2 \pi f C_{\text {mech }}}+4 k_{B} T R_{\text {mech }}} .
\end{aligned}
$$

For the frequency shift modulation technique, the equivalent magnetic noise spectral density is given by

$$
\begin{aligned}
b_{n_{-} \text {demod }}(f) & =\mu_{0} \frac{q_{n_{-} \text {demod }}}{\alpha_{N L M E}^{Q}} \\
& =\frac{\mu_{0} \kappa}{\eta} \sqrt{\frac{4 k_{B} T \tan \left(\delta_{\text {mech }}\right)}{2 \pi f C_{\text {mech }}}+4 k_{B} T R_{\text {mech }}} .
\end{aligned}
$$

By using (13) and (17), we have $\eta \approx 2 \varphi_{m} \kappa$ by hypothesis. So, the equivalent magnetic spectral density is ultimately given by 


$$
b_{n_{-} \text {demod }}(f)=\frac{\mu_{0}}{2 \varphi_{m}} \sqrt{\frac{4 k_{B} T \tan \left(\delta_{\text {mech }}\right)}{2 \pi f C_{\text {mech }}}+4 k_{B} T R_{\text {mech }}} .
$$

Also, by using the corresponding physical parameters ( $c f .(22))$, (28) can be rewritten as

$$
b_{n_{-} \text {de mod }}(f)=\frac{\mu_{0} s_{33}^{N L} d_{33, m}}{s_{33} d_{33, m}^{N L}} \sqrt{4 k_{B} T\left(\frac{s_{33} l \tan \left(\delta_{\text {mech }}\right)}{2 \pi f w t}+\frac{m 2 \pi f_{0}}{Q}\right)} .
$$

So, we find that the EMN noise spectral density, related to the mechanical loss from the interfriction and the viscosity in a laminate, presents a $1 / f$ noise behavior and a white noise behavior, respectively. Moreover, one can notice that the equivalent magnetic noise spectral density is independent of the excitation carrier amplitude, $H_{0}$ as we (and some other groups) have experimentally observed at a certain level of this magnetic field amplitude [19, 20, 38, 39, 40]. The expected EMN noise spectral density behavior due to intrinsic noise sources in ME laminate in these conditions is represented in Fig. 3.

\section{MEASUREMENT AND DISCUSSION}

A ME sensor fabricated with commercial Metglas foils and PZT layers was used for our experiments. The sensor was excited around its first longitudinal mechanical resonance of $26.6 \mathrm{kHz}$ by applying a magnetic field along the longitudinal direction, using a Helmholtz coil in series of a resistance of $100 \Omega$. This Helmholtz coil has a transfer function of $7.78 \mathrm{G} / \mathrm{A}$. Another Helmholtz coil of $5.37 \mathrm{G} / \mathrm{A}$ was used to generate the low frequency reference signal in series with a resistance of $10 \mathrm{k} \Omega$. An analyzer (HP 3562A) was used to measure the output signal transfer functions and noise spectral densities.
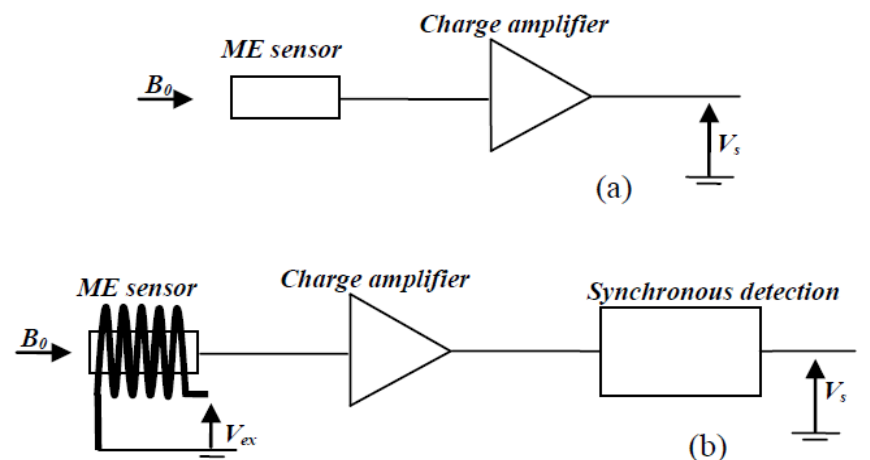

Fig. 4. Sketch view of the (a) passive detection and (b) active detection modes. We notice that $V_{e x}$ induced the modulation field. $B_{0}$ is the sensed signal.

After a classical demodulation process, we measured the transfer function of the ME sensor, which is DC capable, and its equivalent magnetic noise level using this modulation technique. The sketch view of the passive and active detection modes are given in Fig. 4. A magnetic excitation signals $8.25 \mu \mathrm{T}_{\mathrm{rms}}$, were applied on the ME sensor for the modulation detection. We can obtain the transfer function of $6 \mathrm{kV} / \mathrm{T}$ in the low frequency range. The normalized transfer functions at $1 \mathrm{~Hz}$ for classical detection method and modulation technique are

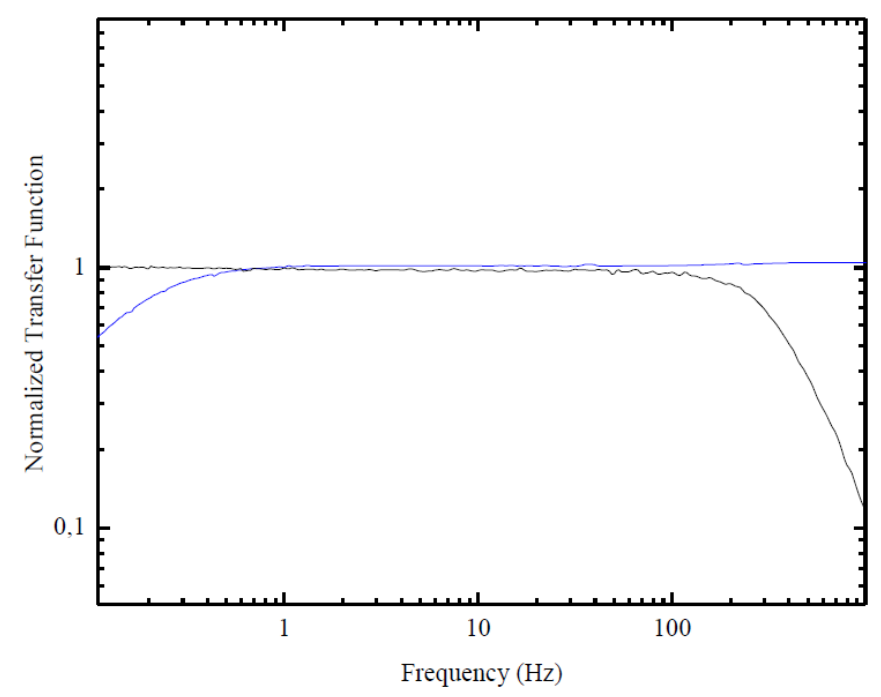

Fig. 5. Experimental transfer function of a ME sensor appearing at low frequencies observed after the demodulation process when using modulation techniques (black curve) and passive mode detection (blue curve). Both curves are normalized by its value at $1 \mathrm{~Hz}$.

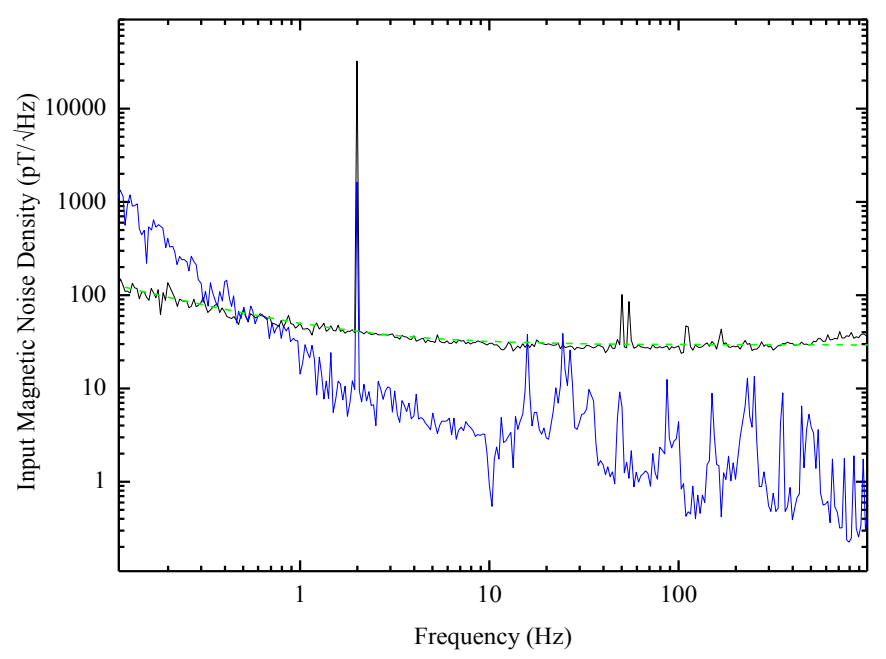

Fig. 6. Experimental equivalent magnetic noise spectral densities of a ME sensor appearing at low frequencies when using modulation techniques (black curve) and passive mode detection (blue curve). The green dashed curve is the simulated curve by using parameters given in table I.

also compared. (cf. Fig. 5).. The equivalent magnetic noise spectral densities for modulation and passive mode detections are given and compared in Fig. 6. We observed that in low frequency (eg. below $0.5 \mathrm{~Hz}$ ) the extrinsic sensor fluctuation can be avoided via the modulation technique. Both the simulation and theoretical EMN level are around $50 \mathrm{pT} / \sqrt{\mathrm{Hz}}$ at $1 \mathrm{~Hz}$.

Table I: Physical, sensors \& material parameters

\begin{tabular}{|c|c|c|c|}
\hline$\mu_{0}$ & $4 \pi 10^{-7} \mathrm{H} / \mathrm{m}$ & $d_{33, m}$ & $6.510^{-10}\left(\mathrm{~T} \mathrm{~m}^{2}\right) / \mathrm{V}$ \\
\hline$t_{m}$ & $150 \mu \mathrm{m}$ & $w$ & $1 \mathrm{~cm}$ \\
\hline$s_{33, m}$ & $9.110^{-12} \mathrm{~m}^{2} / \mathrm{N}$ & $\varphi_{m}$ & $910^{-5} \mathrm{~T} \mathrm{~m}^{2}$ \\
\hline$k_{B}$ & $1.3810^{-23} \mathrm{~J} / \mathrm{K}$ & $T$ & $300 \mathrm{~K}$ \\
\hline $\tan \left(\delta_{\text {mech }}\right)$ & $0.11 \%$ & $C_{\text {mech }}$ & $410^{-7} \mathrm{~m} / \mathrm{N}$ \\
\hline$R_{\text {mech }}$ & $254 \mathrm{~kg} / \mathrm{s}$ & \multicolumn{3}{l}{} \\
$s_{33, m}$ and $d_{33, m}$ are adjusted to fit with the experimental measurement.
\end{tabular}




\section{CONCLUSION}

The effects of temperature induced thermo-magnetic, thermo-electric and thermo-mechanical losses has been analyzed. All of them can be represented as low-frequency intrinsic noise sources in a strain coupled ME laminate composite. The thermo-magnetic noise contribution to the output electric noise can be considered to be negligible. In the passive mode, the thermo-electric noise sources (Johnson noise and dielectric loss noise) appear as the dominant intrinsic noise sources. However, when the magnetoelectric sensor is operated in a modulation mode, low-frequency thermo-mechanical noise will modulate the excitation carrier signal via the mechanical non-linearity of the system and will thus be distributed around the carrier frequency. This noise appears as the dominant noise after the demodulation process when excitation carriers of large amplitude are used, as we and some other groups have observed and investigated, but not well explained yet [19, 20, 38, 39, 40]. From this theoretical analysis, we can conclude that when using a magnetic field modulation on a ME sensor in the longitudinal vibration mode, a weaker mechanical non-linearity $\kappa$ and/or a stronger magnetic non-linearity $\eta\left(\right.$ ratio $\alpha 1 / \varphi_{m}$ ) can lead to a lower equivalent magnetic noise level spectral density as given by $(28,29)$.

\section{REFERENCES}

[1] S. Dong, J. Cheng, J. F. Li et D. Viehland, «Enhanced magnetoelectric effects in laminate composites of Terfenol-D-PbZr,TiO3 under resonant drive», Appl. Phys. Lett. , vol. 83, p. 4812, 2003.

[2] D. V. Chashin, Y. K. Fetisov, K. E. Kamentsev et G. Srinivasan, «Resonance magnetoelectric interactions due to bending modes in a nickel-lead zirconate titanate bilayer», Appl. Phys.Lett., vol. 92, p. 102511, 2008.

[3] J. G. Wan, Z. Y. Li, Y. Wang, M. Zeng, G. H. Wang et J.-M. Liu, «Strong flexural resonant magnetoelectric effect in

Terfenol-D/epoxy- $\mathrm{Pb}(\mathrm{Zr}, \mathrm{Ti}) \mathrm{O} 3$ bilayer», Appl. Phys. Lett. , vol. 86, p. 202504, 2005.

[4] Y. Wang, X. Zhao, W. Di, H. Luo et S. W. Or, «Magnetoelectric voltage gain effect in a long-type magnetostrictive/piezoelectric heterostructure», Appl. Phys. Lett. , vol. 95, p. 143503, 2009.

[5] D. A. Fillippov, V. M. Laletin et G. Srinivasan, «Low Frequency and Resonance Magnetoelectric Effects in Nickel Ferrite-PZT Bulk Composites», Technical Physics, vol. 57, pp. 44-47, 2012.

[6] H.-C. He, J. Ma, Y. Lin et C. W. Nan, «Influence of relative thickness on multiferroic properties of bilayered $\mathrm{Pb}(\mathrm{Zr} 0.52 \mathrm{Ti} 0.48) \mathrm{O} 3-\mathrm{CoFe} 2 \mathrm{O} 4$ thin films», J. Appl. Phys. , vol. 104, p. 114114, 2008.

[7] H. Greve, E. Woltermann, R. Jahns, S. Marauska, B. Wagner, R. Knochel, M. Wutting et E. Quandt, «Low damping resonant magnetoelectric sensors», Appl. Phys. Lett., vol. 97, p. 152503, 2010.

[8] Z. Xing, J. Li et D. Viehland, «Modeling and the signal-to-noise ratio research of magnetoelectric sensors at low frequency», Appl. Phys. Lett., vol. 91, p. 142905, 2007.

[9] X. Zhuang, M. Lam Chok Sing, C. Cordier, S. Saez, C. Dolabdjian, J. Das, J. Gao, J. Li et D. Viehland, «Analysis of Noise in Magnetoelectric Thin-Layer Composites Used as Magnetic Sensors», IEEE Sensors J., vol. 11, p. 2183, 2011.

[10] Y. Wang, D. Gray, D. Berry, J. Gao, M. Li, J. Li et D. Viehland, «An Extremely Low Equivalent Magnetic Noise Magnetoelectric Sensor», Adv. Mater., vol. 23, pp. 4111-4114, 2011.

[11] F. A. Levinzon, «Noise of Piezoelectric Accelerometer With Integral FET Amplifier», IEEE Sensors J. , vol. 5, p. 1235, 2005.

[12] Z. Xing, J. Li et D. Viehland, «Noise and scale effects on the signal-to-noise ratio in magnetoelectric laminate sensor/detection units», Appl. Phys. Lett. , vol. 91, p. 182902, 2007.

[13] F. Li, F. Zhao, Q. M. Zhang et S. Datta, «Low-frequency voltage mode sensing of magnetoelectric sensor in package», Electron. Lett., vol. 46, p. 1132, 2010.

[14] X. Zhuang, M. Lam Chok Sing, S. Saez, C. Cordier, C. Dolabdjian, J. Gao, J. Li et D. Viehland, «Theoretical analysis of the intrinsic magnetic noise spectral density of magnetostrictive-piezoelectric laminated composites», J. Appl. Phys., vol. 109, p. 124512, 2011.

[15] L. Chen, X. M. Xiong, H. Meng, P. Lv et J. X. Zhang, «Migration and redistribution of oxygen vacancy in barium titanate ceramics», Appl. Phys. Lett., vol. 89, p. 071916, 2006.

[16] Z. Wang, R. Zhang, E. Sun et W. Cao, «Contributions of domain wall motion to complex electromechanical coefficients of $0.62 \mathrm{~Pb}(\mathrm{Mg} 1 / 3 \mathrm{Nb} 2 / 3) \mathrm{O} 3-0.38 \mathrm{PbTiO} 3$ crystals», J. Appl. Phys., vol. 107, p. 014110, 2010.

[17] J. Petrie, D. Viehland, D. Gray, S. Mandal, G. Sreenivasulu, G. Srinivasan et A. S. Edelstein, «Enhancing the sensitivity of magnetoelectric sensors by increasing the operating frequency», J. Appl. Phys., vol. 110, p. 124506, 2011.

[18] X. Zhuang, M. Lam Chok Sing, C. Cordier, S. Saez, C. Dolabdjian, L. Shen, J. Li et D. Viehland, «Evaluation of Applied Axial Field Modulation Technique on ME Sensor Input Equivalent Magnetic Noise Rejection», IEEE Sensors J., vol. 11, p. 2266, 2011.

[19] S. M. Gillette, A. L. Geiler, D. Gray, D. Viehland, C. Vittoria et V. G. Harris, «Improved Sensitivity and Noise in Magneto-Electric Magnetic Field Sensors by Use of Modulated AC Magnetostriction», IEEE Mag. Lett., vol. 2, p. 2500104, 2011.

[20] J. R. Petrie, J. Fine, S. Mandal, G. Sreenivasulu, G. Srinivasan et A. Edelstein, «Enhanced sensitivity of magnetoelectric sensors by tuning the resonant frequency», Appl. Phys. Lett., vol. 99, p. 043504, 2011.

[21] M. D. Mermelstein et A. Dandrifge, «Dynamic sensitivity and thermal noise analysis of a magnetoelastic», Appl. Phys. Lett., vol. 51, p. 1640, 1987.

[22] M. B. Welssman, «1/f noise and other slow, noneexponential kinetics in condensed matter», Rev. Mod. Phys. , vol. 60, p. 537, 1988.

[23] T. B. Gabrielson, «Mechanical-Thermal Noise in Micromachined Acoustic and Vibration sensors», IEEE Trans. Electron. Dev., vol. 40, p. 903, 1993.

[24] N. Smith, «Modeling of thermal magnetization fluctuations in thin-film magnetic devices», J. Appl. Phys. , vol. 90, p. 5768, 2001.

[25] L. A. Rocha, E. Cretu et R. F. Wolffenbuttel, «Measuring and interpreting the mechanical-thermal noise spectrum in a MEMS», $J$. Micromech. Microeng., vol. 15, p. S30, 2005.

[26] G. Lawes et G. Srinivasan, «Introduction to magnetoelectric coupling and multiferroic films», J. Phys. D: Appl. Phys., vol. 44, p. 243001, 2011.

[27] H. T. Hardner, M. B. Weissman, M. B. Salamon et S. S. P. Parkin, «Fluctuation-dissipation relation for giant magnetoresistance $1 / \mathrm{f}$ nosie», Phys. Rev; B, vol. 48, p. 16156, 1993.

[28] H. B. Callen et T. A. Welton, «Irreversibility and Generalized Noise», Phys. Rev., vol. 83, p. 34, 1951.

[29] C. S. Ganpule, V. Nagarajan, H. Li, A. S. Ogale, D. E. Steinhauer, S. Aggarwal, E. Williams, R. Ramesh et P. De Wolf, «Role of $90^{\circ}$ domains in lead zirconate titanate thin films», Appl. Phys. Lett., vol. 77, p. 292, 2000.

[30] F. Xu, S. Trolier-McKinstry, W. Ren, B. Xu, Z.-L. Xie et K. J. Hemker, «Domain wall motion and its contribution to the dielectric and piezoelectric properties of lead zirconate titanate films», J. Appl. Phys., vol. 89, p. 1336, 2001.

[31] G. L. Rhun, I. Vrejoiu et M. Alexe, «Piezoelectric response hysteresis in the presence of ferroelastic $90^{\circ}$ domain walls», Appl. Phys. Lett., vol. 90, p. 012908, 2007

[32] Z. Xing, J. Zhai, S. Dong, J. Li, D. Viehland et W. G. Odendaal, «Modeling and detection of quasi-static nanotesla magnetic field variations using magnetoelectric laminate sensors», Meas. Sci. Technol., vol. 103, p. 033903, 2008.

[33] T. H. Stievater, W. S. Rabinovich, H. S. Newman, R. Mahon, P. G. 
Goetz, J. L. Ebel et D. J. McGee, «Measurement of thermal-mechanical noise in microelectromechanical systems», Appl. Phys. Lett., vol. 81, p. 1779,2002

[34] Y. Yao, Y. Hou, s. Dong, X. Huang, Q. Yu et X. Li, «Influence of magnetic fields on the mechanical loss of Terfenol-D/PbZr0.52Ti0.4803/Terfenol-D laminated composites», Journal of Alloys and Compounds, vol. 509, p. 6920, 2011

[35] Z. Diao, E. R. Nowak, G. Feng et J. M. D. Coey, «Magnetic Noise in Structured Hard Magnets», Phys. Rev. Lett. , vol. 104, p. 047202, 2010.

[36] Y. Liu et P. Grutter, «Theory of magnetoelastic dissipation due to domain wall width oscillation», J. Appl. Phys. , vol. 83, p. 5922, 1998.

[37] R. B. Williams, «Nonlinear Mechanical and Actuation Characterization of Piezoceramic Fiber Composites», Virginia polytechnic institute and state university, thesis, 2004.

[38] R. Jahns, H. Greve, E. Woltermann, E. Quandt et R. Knöchel, «Sensitivity enhancement of magnetoelectric sensors through frequency-conversion», Sens. and Actuators A: Phy., vol. 183, pp. 16-21, 2012.

[39] L. Shen, M. Li, J. Gao, Y. Shen, J. F. Li, D. Viehland, X. Zhuang, M. Lam Chok Sing, C. Cordier, S. Saez et C. Dolabdjian, «Magnetoelectric nonlinearity in magnetoelectric laminate sensors», J. Appl. Phys. , vol. 110, p. 114510, 2011

[40] X. Zhuang, GREYC internal report, July 2010.

Xin Zhuang was born in Qingdao, China, in 1983. He received the first B.S. degree in electronic science and technology from the Ocean University of China, Qingdao, and a second B.S. degree in electronics electrical engineering and automation and the M.S. degree in signal and circuits from the University of Brest, Brest, France, in 2007 and 2009, respectively. Since 2009 he has been with the Department of Electronics at GREYC Laboratory, ENSICAEN and the University of Caen Lower Normandy, Caen, France, where he received his Ph.D. degree in electronics and instrumentation. He is currently working as a post-doc fellow at GREYC Laboratory performance optimization of magnetic sensors.

His main research focus is on the performance of magnetoelectric laminated composites.

Marc Lam Chok Sing received the Engineer degree from the Ecole Nationale Supérieure d'Ingénieurs de Caen (ENSICAEN), France, in 1985 and the Ph.D. degree in Science from the University of Caen, Caen, France, in 1989.

$\mathrm{He}$ is currently a Lecturer in Electronics at the ENSICAEN School of Engineering. His present research interests include magnetic sensors, high-resolution magnetometers and low-noise electronics.

Christophe Dolabdjian was born in Enghien-les-Bains, France, in 1967. He received the M.S. and the Ph.D. degrees in electronics and instrumentation from the University of Caen, Caen, France, in 1991 and 1994, respectively, and the Habilitation Diploma in 2000.

In 1994, he joined the Groupe de Recherche en Informatique, Image, automatique et Instrumentation de CAEN CNRS UMR 6072 of ENSICAEN and the University of CAEN as an Assistant Professor, where he has been a Professor of Electronics, since 2001. His research interests included studied, development, optimization, improvement and comparison of numerous very high sensitivity and very low magnetic noise sensors (SQUID, JFM, Flux-gate, GMR, GMI, $\mu$ Hall, Hybrid...), as well as their integration in applications, in open or close environment, like Biomagnetism and Non-Destructive Testing. He was also an Assistant Director and Director of the Doctoral School "SIMEM" from 2002 to 2006 and from 2007 to 2009, respectively. Presently, he is the Head of the Electronic Team of the GREYC until 2007 and in charge of the "Licence Pro MCA" professional B.A. of the UCBN until 2001.

Peter Finkel is a Materials scientist and R\&D Scientist in the Devices, Sensors and Materials R\&D Branch at the Naval Undersea Warfare center (NUWC) in Newport, RI. Peter received a Ph.D. degree in materials science/low temperature physics from Drexel University, a master's degree in physics from Queens College at The City University of New York. His research areas include experimental solid-state physics, magnetism, and materials science, with a focus on sensors, ultrasonics, and spectroscopy. His work in the transduction materials group at NUWC concentrates on single-crystal piezoelectric materials used in acoustic devices and novel magnetoelectric sensors. Prior to joining Drexel, Dr. Finkel was a Physicist and Research Member of the Technical Staff at the RAC/GE/Thomson R\&D Center, Lancaster, Pa. He has authored more than 35 refereed publications and has delivered many invited lectures and seminars.

Jiefang Li received her Ph.D. degree in solid-state science from The Pennsylvania State University. She is currently a research professor of Materials Science and Engineering at Virginia Tech. Her research interests include ferroelectric, piezoelectric, dielectric, and magnetoelectric materials. She has been instrumental in the development and study of magnetoelectric laminate composites. Jiefang has published more than 100 peer-reviewed journal articles.

Dwight Viehland is currently in the Department of Materials Science and Engineering at Virginia Tech. He received B.S. and M.S. degrees from the University of Missouri-Rolla, and a Ph.D. from The Pennsylvania state University. Dwight is an experimental solid-state scientist in the structure and properties of condensed matter and thin layers. His research focuses on sensor materials including magnetoelectricity, piezoelectricity, and magnetostriction. Since joining Virginia Tech, the Viehland laboratory began a new area of research that involved the development of novel materials and composites with large magneto-electric exchanges. 